\title{
Construcción de conocimiento en educación virtual: Nuevos roles, nuevos cambios
}

\section{Knowledge Construction in Virtual Education: New Roles, New Approaches}

\author{
Cristina Hennig Manzuoli \\ Centro de Tecnologías para la Academia. Universidad de La Sabana. Colombia. \\ cristina.hennig2@unisabana.edu.co \\ Anna Escofet Roig \\ Universidad de Barcelona. España. \\ anna.escofet@ub.es
}

\begin{abstract}
Resumen
El presente artículo expone los resultados de un estudio que tuvo como propósito analizar las percepciones de los profesores frente a su rol en entornos digitales de enseñanza y aprendizaje en Colombia, Suramérica, realizado en dos instituciones de educación superior que ofrecen programas de pregrado en modalidad virtual. La investigación empleó técnicas cualitativas y cuantitativas mediante la realización de grupos focales y observación de aulas virtuales, también se realizó una encuesta a docentes con experiencia en la modalidad de más de seis meses. Los resultados sugieren que el acompañamiento del profesor y la comunicación entre estudiantes son escasos, así como dificultades en la interacción que no permiten un verdadero trabajo colaborativo que esté enfocado a la construcción de conocimiento.
\end{abstract}

\section{Palabras claves}

Construcción de conocimiento, E-learning, Educación virtual, Rol del tutor, Rol del estudiante

\begin{abstract}
This paper presents the results of a study that analyzes the perceptions of teachers facing their role in digital teaching and learning environments in Colombia, South America. The study was carried out in two Higher Education institutions that offer undergraduate programs in virtual mode. The research used qualitative and quantitative techniques by conducting focus groups and observation of virtual classrooms. A survey was also conducted among teachers with more than six-month experience in the modality. The results suggest that the teacher role by the student and the communication between students are scarce, and that difficulties in the interaction do not allow a true collaborative focused on the construction of knowledge.
\end{abstract}

\section{Key words}

Knowledge construction, E-Learning, Virtual education, Tutor's role, Student's role

\section{Introducción}

Tanto en la modalidad presencial como en la virtual la finalidad es la construcción de conocimiento por parte de los estudiantes. Diferentes estudios (Sorathia y Servidio, 2012; Naykii, et al, 2008; Barbera, 2006) han profundizado en el tema de la elaboración de conocimiento por parte de los aprendices, los hallazgos principalmente han estado enfocados a la importancia de la interacción y la realimentación en ambientes virtuales de aprendizaje. Los 
procesos de comunicación entre estudiantes y de éstos con el profesor, impulsan la formación de los estudiantes.

La interacción por tanto juega un papel fundamental en el proceso de enseñanza - aprendizaje de los estudiantes. Esta interacción entendida como proceso de comunicación, contribuye a la apropiación del conocimiento, a través del seguimiento y realimentación que el profesor realiza a los avances de los estudiantes con las actividades de aprendizaje propuestas, en palabras de Barbera, 2008, la comunicación en el triángulo interactivo profesor, estudiante, contenidos promueve la construcción de conocimiento por parte de los aprendices.

La formación del profesor por tanto es fundamental para la mediación que éste realiza en el proceso de aprendizaje de los estudiantes, los estudios de Ling y Sing (2014), encuentran diferencias en las percepciones de los profesores para la implementación del modelo TPACK, luego de un curso de formación encaminado a la integración pedagógica de las TIC. Por su parte, los estudios de Sang et al (2010) encuentran que los profesores formados en la corriente constructivista son más proclives al uso efectivo de las tecnologías de la información y la comunicación en sus clases. El razonamiento pedagógico del profesor influye en el nivel de integración de TIC que logre en sus clases, así como la elaboración de un plan de integración pedagógica de las TIC que privilegie la construcción de conocimiento por parte de los estudiantes (Hammond, Reynolds \& Ingram, 2011).

Los profesores formados para la integración pedagógica de las TIC mediante el uso de Blog (Goktas Demirel, 2012) encuentran que la aplicación de la capacitación recibida, a través de herramientas tecnológicas, les permitió la práctica en el uso de las TIC y una actitud positiva frente al uso de las mismas.

Los desafíos para los profesores son grandes, dado que, requieren de procesos de reflexión para identificar el potencial que tienen las herramientas tecnológicas para el cumplimiento de las metas de aprendizaje de cada curso. La preparación de las ayudas educativas que el profesor realiza para el proceso de aprendizaje de los estudiantes, deben garantizar que se promueva la mediación de la actividad constructiva del estudiante, en palabras de Onrubia y Mauri (2008), mediación que realiza el profesor con apoyo de las TIC y que tiene en cuenta el grado de ajuste de la actividad conjunta.

El acompañamiento del profesor por tanto marca las posibilidades de una verdadera construcción de conocimiento en entornos virtuales de aprendizaje. Este nuevo rol del profesor implica que se desplaza en el proceso, situándose al lado del estudiante para proporcionarle las orientaciones necesarias, con el fin de alcanzar las metas de aprendizaje propuestas. Los profesores expertos prestan más acompañamiento a los estudiantes en el trabajo colaborativo que desarrollan con sus compañeros que aquellos profesores que se encuentran en proceso de formación (Kopp et al 2012).

Por tanto se hace necesario indagar acerca del rol del profesor en modalidad virtual, en cuanto a la contribución de sus orientaciones que permitan una verdadera construcción de conocimiento en educación virtual, éste es el propósito principal de la investigación realizada con un grupo de 28 profesores pertenecientes a dos instituciones de pregrado de modalidad virtual en Bogotá, Colombia.

\section{Método}

Las características y objetivos de esta investigación nos llevan a optar por un enfoque investigativo que privilegia los métodos cualitativos. Aunque se da inicio a la investigación con la aplicación de una encuesta, los resultados de la misma se utilizaron para profundizar a través de instrumentos cualitativos (grupos focales y observación). Se pretende con la investigación 
ahondar en las percepciones de los profesores frente a su rol en educación virtual. Se busca por tanto entender una situación particular que permita proponer alternativas frente a la importancia del rol del tutor en modalidad virtual.

El proyecto se inscribe en un enfoque mixto, dado que se utilizarán encuestas y grupos focales con profesores y observación de las aulas virtuales que se usan para formación de los estudiantes. El análisis cuantitativo a través de las encuestas permitió conocer las percepciones de los profesores, frente su rol en entornos digitales de enseñanza y aprendizaje. El análisis cualitativo a través de la observación y los grupos focales por su parte, permitió profundizar en aquellos aspectos que se encontraron relevantes en el análisis de la encuesta aplicada.

El estudio es de tipo descriptivo, ya que se pretende caracterizar las percepciones del profesor frente a su rol en educación virtual. Los estudios descriptivos buscan especificar las propiedades importantes de personas, grupos, comunidades o cualquier otro fenómeno que sea sometido a análisis. Evalúan diversos aspectos, dimensiones o componentes del fenómeno o fenómenos sociales a investigar. Se presenta entonces como limitación en estos estudios la posibilidad de generalización, dado que pretenden dar a conocer las características de un fenómeno o hecho en particular.

Para Ander Egg (1987), los estudios descriptivos tienen como objetivo identificar las características de personas, grupos, comunidades o cualquier otro fenómeno que sea sometido a análisis, es decir, buscan conocer quién, dónde, cuándo, cómo y por qué del sujeto de estudio, y principalmente evalúan diversos aspectos, dimensiones o componentes del fenómeno o fenómenos a investigar. En un estudio descriptivo se seleccionan una serie de cuestiones y se mide cada una de ellas independientemente, para así describir lo que se investiga. La investigación descriptiva requiere de un considerable conocimiento del área que se investiga para formular las preguntas específicas que busca responder, se basa en la evaluación de uno o más atributos del fenómeno descrito. Pueden ofrecer la posibilidad de predicciones rudimentarias.

\subsection{Participantes}

Los participantes de la investigación fueron 28 profesores que se desempeñan como tutores virtuales en programas de pregrado con 80 o $100 \%$ de virtualidad, cuya experiencia en este ejercicio supera los seis meses para garantizar que conocen las dinámicas que se generan en esta modalidad de educación. Los profesores pertenecen a dos instituciones de educación superior de carácter privado que ofrecen programas de pregrado en modalidad virtual de las cuales se protege su identidad por razones de confidencialidad de los datos. La institución 1 cuenta con un programa de pregrado y cuatro programas de especialización en modalidad virtual con alrededor de 3000 estudiantes de acuerdo con los datos suministrados por el Sistema Nacional de Información de Educación Superior del Ministerio de Educación Nacional de Colombia, para esta modalidad cuenta con 18 profesores, la institución 2 cuenta con cinco programas de pregrado y dos especializaciones en modalidad virtual con un total de 4106 estudiantes.

Los profesores de la institución 1 tienen estudios de pregrado en programas como: Ingeniería de Sistemas, Ingeniería Industrial, Logística, Administración de Empresas, Ingeniería de Telecomunicaciones, Cocina, Psicología, Derecho, Biología, Filosofía, Comercio internacional, Contaduría pública, Administración Pública y Fisioterapia y especializaciones y máster en: Telemática, Redes sociales y aprendizaje digital, Business Administration, Gerencia de proyectos, Gerencia de talento humano, Derecho laboral y Relaciones industriales, Neurogenética, Gerencia de Marketing, Educación, Gerencia en Logística, Salud ocupacional, Políticas públicas, Gerencia estratégica de alimentos y bebidas. 
Los profesores de la institución 2, tienen estudios de pregrado en programas como: Ingeniería de Sistemas, Ingeniería Industrial, Logística, Administración de Empresas, Ingeniería de Telecomunicaciones, Psicología, Derecho, Biología, Filosofía, Comercio internacional, Contaduría pública, Administración Pública y Fisioterapia y especializaciones y máster en: Business administración, Gerencia de proyectos, Gerencia de talento humano, Derecho laboral y Relaciones industriales, Gerencia de Marketing, Educación, Gerencia en Logística, Salud ocupacional, Políticas públicas.

\subsection{Recolección de datos}

Se utilizaron tres instrumentos de recolección de datos:

-Encuesta en línea sobre los usos de las TIC que hacen los profesores y las creencias que los mismos tienen sobre la tecnología. Este instrumento fue validado por expertos, quienes hicieron recomendaciones para mejorar la comprensión de las preguntas por parte de los profesores. La encuesta presenta tres partes (1) Información general sobre los participantes. (2) Usos de las TIC por parte de los profesores y (3) Actitudes de los profesores hacia la tecnología.

-Observación no participante de aulas virtuales se rastrearon los cursos de los profesores, con el fin de tomar notas de observación sobre las dinámicas de trabajo en las actividades de aprendizaje propuestas.

-Grupos focales con los profesores participantes en la encuesta en los que se profundizó sobre las percepciones de los mismos, frente al trabajo que se desarrolla con los estudiantes a través de la mediación de recursos y herramientas tecnológicas.

\subsection{Análisis de datos}

En análisis de los datos se realizó mediante la teoría fundamentada, es decir que se extrajo información de los datos recolectados con los instrumentos propuestos en el estudio (encuesta en línea, observación de aulas virtuales y grupos focales). Los datos de la encuesta se procesaron en el programa SPSS; las observaciones y grupos focales en el programa Atlas ti, con el análisis de los datos se desarrollaron categorías de análisis que emergieron de los datos mediante codificación abierta, codificación axial y codificación selectiva.

\section{Hallazgos}

\subsection{Resultados cuantitativos}

Se procesaron los datos para obtener resultados tanto de estadística descriptiva (conteos de frecuencias tanto en número como porcentaje), como de estadística inferencial (tablas de contingencia y chi cuadrado), los resultados cuantitativos muestran que los profesores utilizan diferentes herramientas tecnológicas para mediar las actividades de aprendizaje que desarrollan con los estudiantes, se evidencia el uso frecuente de foros de discusión principalmente para las actividades relacionadas con el trabajo grupal, para la indagación de temáticas, el intercambio de información y la discusión de temas los foros de discusión se utilizan para ejercicios de trabajo colaborativo como se muestra en la gráfica I.

Gráfica I. Use tools for collaborative work. 


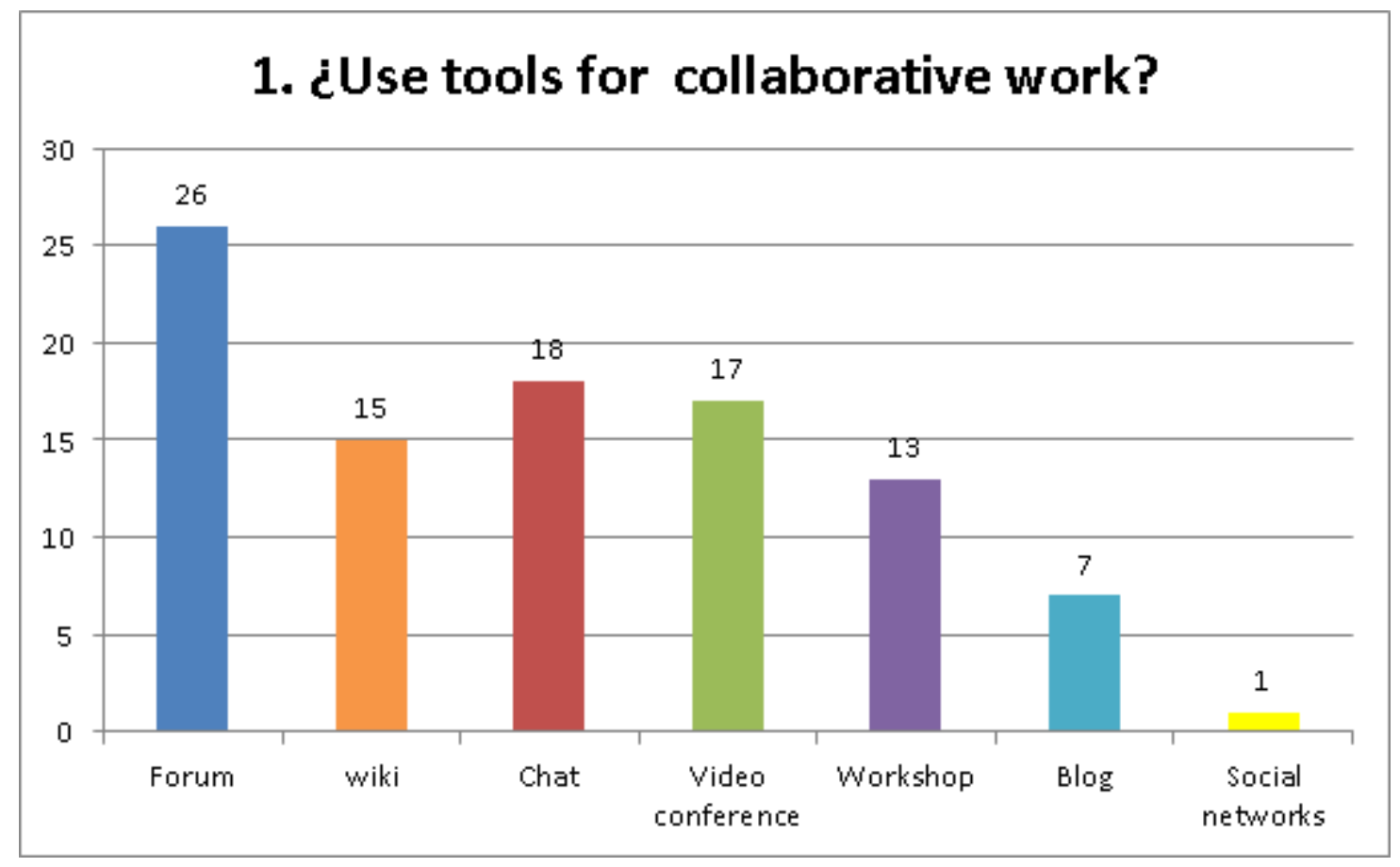

Los profesores son conscientes de las posibilidades que ofrecen las TIC en educación, creen que pueden usar las TIC en sus clases con eficacia, fomentan la comunicación entre estudiantes y de estos con los contenidos, a través de herramientas tecnológicas y creen que la enseñanza con tecnología es eficaz. En términos generales los profesores tienen actitudes positivas frente al uso de tecnología en educación.

Los profesores que creen que las TIC los hace más eficaces en sus clases y creen que el uso de tecnología es eficaz utilizan diferentes herramientas tecnológicas para la discusión de temas con los estudiantes.

\subsection{Resultados cualitativos}

Los principales hallazgos cualitativos están relacionados con las categorías motivación, diseño de los cursos y rol del profesor:

Categoría motivación:

Se encontró que el acompañamiento es "vital" para la construcción de conocimiento en esta modalidad de educación y que la escasa interacción entre estudiantes limita la elaboración de conocimiento de los mismos en las actividades de trabajo colaborativo que se proponen. El papel del profesor debe estar enfocado a brindar orientaciones para el trabajo de los estudiantes, así como propiciar la comunicación entre estudiantes y de estos con los contenidos de aprendizaje.

“...creo que el acompañamiento es vital, si bien la estrategia pedagógica es el elemento fundamental para el desarrollo de la estrategia colaborativa, creo que el acompañamiento docente puede motivar, la gestión del docente al interior del curso" Institución 1, Profesor 6.

"Se acompaña al estudiante durante todo el tiempo que dure el curso, resolviendo inquietudes de carácter académico así como técnico (manejo de la plataforma) Se está pendiente de formular las diversas actividades así como su revisión, calificación y retroalimentación" Institución 2, profesor 3. 
Otros hallazgos están relacionados con la relevancia de las orientaciones del profesor para mediar el proceso de enseñanza - aprendizaje, tal como las actividades, tanto de trabajo individual como colaborativo principalmente en la modalidad virtual. Los profesores proponen diferentes actividades de aprendizaje con mediación de la tecnología, por ejemplo, para el desarrollo de la argumentación escrita pero no han encontrado resultados positivos principalmente porque los estudiantes se dividen el trabajo y no se da una verdadera interacción para trabajo en equipo.

“...usted busque esto y yo busco esto y arman un engendro, no puedo decirlo de otra manera, arman lo que pueden mejor dicho” Institución 1, profesor 1.

Los profesores ofrecen actividades de aprendizaje como wiki, foros, blogs, twitter, sin que se dé una verdadera construcción de conocimiento por parte de los estudiantes, pues los estudiantes hacen uso de todas las herramientas tecnológicas como foro de discusión, sin tener en cuenta las contribuciones individuales para realizar un verdadero trabajo colaborativo.

"Cuando viene el proceso de generar de hacer algo con toda esa información que se maneja, vienen los descalabros porque realmente yo no encuentro mucha articulación pues la gente participa pero ya en el momento de enviar participación al foro, realmente son muy limitadas las participaciones o la construcción que yo noto que se hace del conocimiento en el tema" Institución 1, profesor 2

"Se incentiva la interacción a través de los aportes constructivos, por ejemplo, en un foro, parte del diseño instruccional está dado, en buscar que entre los estudiantes hagan aportes a otros, a partir del trabajo realizado, pero es importante que estos aportes se tomen como un insumo para fortalecer la producción académica antes de la entrega final. Se incentiva a que los estudiantes compartan sus conocimiento, más allá de realizar una tarea o cumplir con un trabajo, la intención es que entre todos hagan un especial aporte a la gestión del conocimiento" Institución 2, profesor 2.

Categoría diseño de los cursos:

Los profesores manifiestan que ofrecen a los estudiantes actividades de aprendizaje enfocadas a la construcción de conocimiento pero que las participaciones de los estudiantes son limitadas y no se da argumentación en los ejercicios propuestos. Sin embargo consideran los profesores que los estudiantes mayores realizan mejores contribuciones al trabajo colaborativo que se desarrolla con ellos.

“...pues la gente participa pero ya en el momento de enviar participación al foro, realmente son muy limitadas las participaciones o la construcción que yo noto que se hace del conocimiento en el tema" Institución 1, profesor 2.

“...alguna otra oportunidad hay estudiantes que sobre todo en las asignaturas de carreras virtuales son estudiantes un poco mayores que ya tienen una formación como tú lo decías hace unos momentos, uhm ellos si ya generan un poco más el debate, si con base no solo en las preguntas detonantes sino con el material multimedia que se genera para ellos entonces ese es otro escenario, ellos si generan un poco de debate y construyen un poco más de conocimiento entre todos" Institución 1, profesor 3.

\section{Categoría rol del profesor:}

Consideran los profesores que las orientaciones del profesor con claves para que se dé un verdadero proceso formativo que enriquezca la construcción de conocimiento. 
"Creo que el acompañamiento es vital, si bien la estrategia pedagógica es el elemento fundamental para el desarrollo de la estrategia colaborativa, creo que el acompañamiento docente puede motivar, la gestión del docente al interior del curso, si uno cuelga un foro en el que todos tienen que participar y construir entre ellos mismos y lo abandonan es complicado que el trabajo colaborativo se dé, porque evidentemente no conocen el tema" Institución 1, profesor 6.

"Se debe atender cualquier solicitud de los estudiantes, como prioridad. Las llamadas telefónicas deben atenderse con información puntual y no con largas. De igual forma, los correos. Deben contestarse antes de 24 horas, dando solución a los requerimientos" Institución 2, profesor 1 .

"El monitoreo constante implica la comunicación efectiva, tanto a partir de las participaciones de los estudiantes, pero igualmente se establece comunicación cuando no se evidencia la participación. La retroalimentación de la producción académica es fundamental, para que los estudiantes hagan conciencia de su proceso de construcción de conocimiento. Se retroalimenta ante de publicar una calificación”. Institución 2, profesor 2.

Los profesores proponen diversas actividades de aprendizaje para propiciar el trabajo colaborativo entre estudiantes, sin embargo, considero que difícilmente se da una verdadera construcción de conocimiento, dado que, los estudiantes se limiten a dividirse el trabajo grupal y luego tratan de entregar un único informe con los aporte de todos pero que carece de coherencia y cohesión.

"muchas veces Yo he tenido experiencias como docente en el que los estudiantes están tan viciados en el uso de los foros, que cogen un wiki y lo hacen como un foro, entonces Yo le complemento el aporte de arriba hacia abajo y los demás hacen la misma dinámica del foro en un recurso wiki, entonces en ese momento se pierde el potencial del recurso y precisamente es por falta de concepto, no hay un trabajo previo, estoy hablando desde ... no hay un trabajo de conceptualización en el que se pueda decir mire este recurso tiene estas características y se puede usar para hacer colaboración de tales formas" Institución 1, profesor 1.

“...usted busque esto y yo busco esto y arman un engendro, no puedo decirlo de otra manera, arman lo que pueden mejor dicho" Institución 1, profesor 1.

“...si el docente está pendiente y ve que hay muchos aportes de todos, él puede motivar para que pero porque no opina sobre el comentario del otro, los invito a dar un comentarios constructivo, es otro elemento que nosotros utilizamos. Entonces Yo creo que la gestión del docente es fundamental para motivar el trabajo colaborativo" Institución 1, profesor 6.

"Todo empieza con un gran entusiasmo hasta que les empiezo a hablar de dialéctica. Luego como vamos a construir nuestras intervenciones a partir de esa dialéctica, como vamos a aportar, entonces comienzan los descalabros, todos empiezan muy entusiasmados, abren su cuenta en twitter, empiezan a seguirme nos volvemos amigos y de un momento a otro seguidores o seguidos y cuando viene el proceso de generar de hacer algo con toda esa información que se maneja, vienen los descalabros porque realmente yo no encuentro mucha articulación pues la gente participa pero ya en el momento de enviar participación al foro, realmente son muy limitadas las participaciones o la construcción que yo noto que se hace del conocimiento en el tema" Institución 1, profesor 2.

\section{Discusión}

El estudio indaga sobre las percepciones de los profesores frente a su rol en educación virtual y los usos de las TIC que hacen estos profesores, los resultados sugieren que los profesores tienen actitudes positivas frente a la integración pedagógica de las tecnologías de la información y la comunicación. En los resultados cualitativos se encuentra que los profesores reconocen la importancia del acompañamiento en el proceso formativo de los estudiantes y que estas 
orientaciones en cierta medida contribuyen a la construcción de conocimiento por parte de los mismos. Estos resultados son consistentes con investigaciones previas en las que los hallazgos han estado enfocados al acompañamiento que involucra de acuerdo con Goodyear, Salmon, Spector, Steeples \& Tickner (2001), roles del profesor como facilitador, guía, investigador, tecnológico, administrador y diseñador. Estos papeles desempeñados por el profesor requieren del enriquecimiento de la práctica pedagógica, que le permita planificar actividades de aprendizaje encaminadas a la construcción de conocimiento, en las cuales el profesor se sitúa al lado del estudiante, con el fin de realizar seguimiento y proporcionar realimentación en el proceso de formación.

De la mano del acompañamiento que brinda el profesor a los estudiantes en el proceso formativo se encuentra la interacción que el tutor debe facilitar para establecer mecanismos de comunicación entre estudiantes y de éstos con el profesor, con el fin de realizar el seguimiento y la realimentación oportuna que permita identificar aspectos a mejorar y reforzar aquellos elementos que se ha detectado como positivos (Sorathia \& Servidio, 2012). La experiencia del profesor influye en las orientaciones que el mismo ofrece a los estudiantes, estos hallazgos son acordes con investigaciones previas en las cuales los profesores expertos brindan mayores posibilidades de acompañamiento a los estudiantes Kopp et al (2012).

\section{Conclusiones e implicaciones}

Los profesores reconocen que el acompañamiento al proceso de formación de los estudiantes es vital para la construcción de conocimiento, el acompañamiento involucra de acuerdo con Goodyear, Salmon, Spector, Steeples \& Tickner (2001), roles del profesor como facilitador, guía, investigador, tecnológico, administrador y diseñador.

Otras actividades que debe desarrollar el profesor para cumplir a cabalidad con su rol en el acompañamiento de los estudiantes de acuerdo con Zabalza (2006), son la organización de los espacios de trabajo de los estudiantes en los cuales se tienen en cuenta los intereses y motivaciones de los mismos, así como las posibilidades metodológicas que orienten el desarrollo de las actividades de aprendizaje, tanto individuales como grupales.

Los profesores ofrecen herramientas tecnológicas para el trabajo colaborativo entre estudiantes pero éstos lo entienden como trabajo en grupo en el que se reparten el trabajo luego unifican y entregan un único documento sin coherencia y cohesión. No se evidencia una verdadera interacción entre estudiantes que permita construir conocimiento. En este sentido, los estudios previos (Kopp et al ,2012) encuentran que la experiencia del tutor influye en la orientación que brindan a los estudiantes para el trabajo colaborativo, los tutores con experiencia ofrecen mayor acompañamiento a los estudiantes para facilitar la interacción entre los mismos, que aquellos que tienen escasa experticia.

Las experiencias previas del profesor con el uso de la tecnología parecen también influir en las actitudes de los mismos hacia la integración futura de las TIC en el currículo. En este sentido, la investigación desarrollada por Hismanoglu (2012), encuentra que los profesores con formación y experiencias positivas frente a la tecnología la integran de manera efectiva en sus clases, con miras a mejorar el proceso de enseñanza - aprendizaje de los estudiantes.

Las creencias de los profesores hacia la tecnología también parecen influir en el uso de las TIC que hace el profesor para mediar el proceso de aprendizaje con los estudiantes. Los estudios realizados por Cullen y Greene (2011), pretenden establecer la relación entre las motivaciones y las actitudes de los profesores hacia la tecnología y la integración que realizan en sus clases, encuentran que las creencias de los profesores son predictores fuertes sobre el uso de las TIC que llevan a cabo los mismos. 
Por su parte Mishra y Koehler (2006), enfatizan en la formación de los profesores y la experiencia que han tenido con la tecnología en sus clases. De acuerdo con estos autores la formación en competencias metodológicas, así como tecnológicas le permiten al profesor establecer relaciones entre los aspectos pedagógicos metodológicos y la selección de recursos tecnológicos apropiados para las metas de aprendizaje que se propone con sus estudiantes.

Artículo finalizado el 17 de febrero de 2015

Henning, C. y Escofet, A. (2015). Construcción de conocimiento en educación virtual: Nuevos roles, nuevos cambios. RED, Revista de Educación a Distancia. 45(5). Consultado el (dd/mm/aaaa) en http://www.um.es/ead/red/45

\section{Referencias}

Ander Egg, E. (1987). Métodos y técnicas de investigación social. México: El Ateneo.

Barbera, E. (2006). Psychology and educational science studies. Barcelona: Universitat Oberta de Catalunya.

Barbera, E. (2008). Cómo valorar la calidad de la enseñanza basada en las TIC. Barcelona: Graó.

Cullen, T; Greene, B (2011). "Preservice teachers' beliefs, attitudes and motivation about technology integration". J. Educational computing research, Vol. 45(1) 29-47.

Goktas. Y., Demirel, T. (2012). "Blog-enhanced ICT courses: Examining their effects on prospective teachers". Computers and Education 58, 908-917.

Goodyear, P., Salmon G., Spector, M., Steeples, C y Tickner, S. (2001). "Competence for online teaching: A special report. Educational Technological". Research and Development, 49(1), 65-72.

Hammond, M. Reynolds, L. \& Ingram, J. (2011). "How and why do student teachers use ICT?" Centre for New Technologies Research in Education, Institute of Education, University of Warwick, Coventry CV4 7AL, UK Blackwell Publishing Ltd Journal of Computer Assisted Learning, 27, 191-203.

Hismanoglu, M. (2012). "Prospective EFL Teachers' Perceptions of ICT Integration: A Study of Distance Higher Education in Turkey". Educational Technology and Society, 15 (1), 185196.

Kopp, B et al (2012). "E-tutorial support for collaborative online learning: An explorative study on experienced and inexperienced e-tutors". Computers \& Education 58, pp. 12-20.

Ling, J., Sing, Ch. (2014). "Teacher clusters and their perceptions of technological pedagogical content knowledge (TPACK) development through ICT lesson design". Computers \& Education. 70, 222-232. 
Mishra, P y Koehler, M. (2006). "Technological Pedagogical Content Knowledge: A Framework for Teacher Knowledge". Teachers College Record Volume 108, Number 6, pp. $1017-1054$.

Naykii, P. y otros (2008). "How pictorial knowledge representation mediate collaborative knowledge construction groups". Finlandia: Universidad de Oulu.

Onrubia, J., Mauri, T (2008) En psicología de la educación virtual. Madrid: Morata.

Sang G., Valcke M., Braak J. \& Tondeur J. (2010) "Student teachers' thinking processes and ICT integration: predictors of prospective teaching behaviors with educational technology". Computers \& Education 54, 103-112.

Silva, A. (2008) "Currículo por Proyectos en Educación Virtual. El diseño de los ciclos propedéuticos". Revista Cognición No 13.

Sorathia, K; Servidio, R. (2012). "Learning and Experience: Teaching Tangible Interaction \& Edutainment. International educational technology". Procedia - Social and Behavioral Sciences.

Zabalza, M (2006). Competencias docentes del profesorado universitario. Calidad y desarrollo profesional. Madrid: Narcea. 